
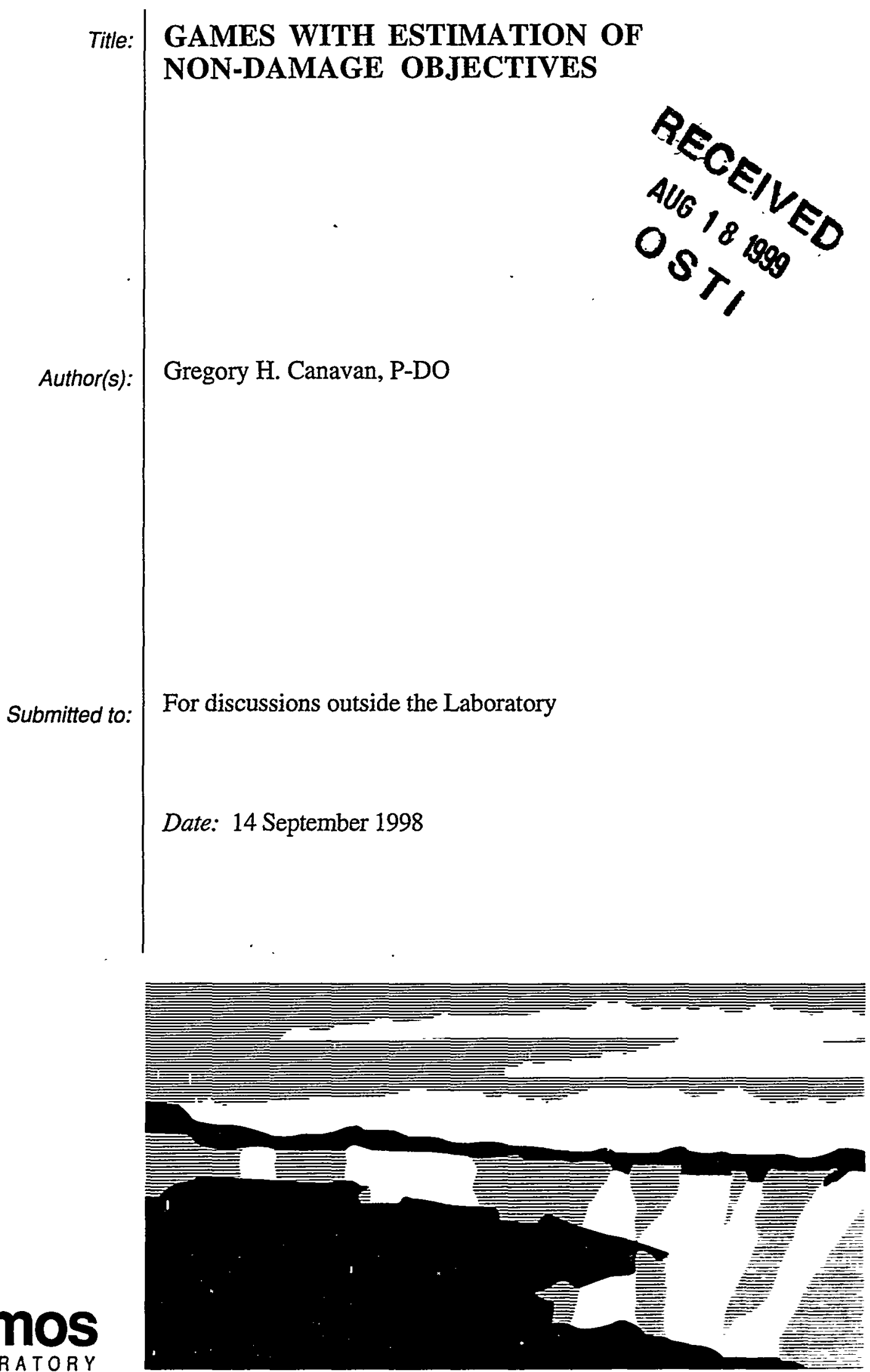

Los Alamos National Laboratory, an affirmative action/equal opportunity employer, is operated by the University of California for the U.S. Department of Energy under contract W-7405-ENG-36. By acceptance of this article, the publisher recognizes that the U.S. Government retains a nonexclusive, royalty-free license to publish or reproduce the published form of this contribution, or to allow others to do so, for U.S. Government purposes. The Los Alamos National Laboratory requests that the publisher identify this article as work performed under the auspices of the U.S. Department of Energy. 


\section{DISCLAIMER}

This report was prepared as an account of work sponsored by an agency of the United States Government. Neither the United States Government nor any agency thereof, nor any of their employees, make any warranty, express or implied, or assumes any legal liability or responsibility for the accuracy, completeness, or usefulness of any information, apparatus, product, or process disclosed, or represents that its use would not infringe privately owned rights. Reference herein to any specific commercial product, process, or service by trade name, trademark, manufacturer, or otherwise does not necessarily constitute or imply its endorsement, recommendation, or favoring by the United States Government or any agency thereof. The views and opinions of authors expressed herein do not necessarily state or reflect those of the United States Government or any agency thereof. 


\section{DISCLAIMER}

Portions of this document may be illegible in electronic image products. Images are produced from the best available original document. 


\title{
GAMES WITH ESTIMATION OF NON-DAMAGE OBJECTIVES
}

\author{
Gregory H. Canavan
}

Games against nature illustrate the role of non-damage objectives in producing conflict with uncertain rewards and the role of probing and estimation in reducing that uncertainty and restoring optimal strategies.

This note discusses two essential elements of the analysis of crisis stability omitted from current treatments based on first strike stability: the role of an objective that motivates conflicts sufficiently serious to lead to conflicts, and the process of sequential interactions that could cause those conflicts to deepen. Games against nature illustrate role of objectives and uncertainty that are at the core of detailed treatments of crisis stability. These models can also illustrate how these games processes can generate and deepen crises and the optimal strategies that might be used to end them. This note discusses two essential elements of the analysis of crisis stability that are omitted from current treatments based on first strike stability: a non-damage objective that motivates conflicts sufficiently serious to lead to conflicts, and the process of sequential tests that could cause those conflicts to deepen. The model used is a game against nature, simplified sufficiently to make the role of each of those elements obvious.

A simple game with a given threshold illustrates the mathematical treatment of the expected gains, losses, and net value. Extension to an unknown maximum threshold shows how the expected value shifts. It illustrates that for any given value and threshold, there is an optimal strategy that maximizes the return to the player, but that the maximum payoff decreases and shifts to larger commitments as the threshold increases, which means that this strategy could take a very large investment and return comparatively little

That can be improved if the contestant can gain information about the payoff from the results of a limited number of trials. Efficient estimation can enable one to cope with threshold uncertainty that would overwhelm non-estimating strategies. The form of the expected loss and gain curves also suggest the possibility of inferring the threshold and value from a small number of trials, but the model suggests that it might be necessary to use exchanges about half as large as the optima to obtain reliable estimates.

Trajectories starting at large threshold uncertainties produce negative returns until the contestant commits significantly. That reduces the variance in the estimate such that the expected value quickly becomes positive, and rejoins the trajectory for small thresholds, albeit at a much lower expected value. A 30-fold increase in roughly doubles the value of commitment at which the trajectories join and reduces the value at that point by a tolerable amount. 
The model does not explicitly contain some features that are included in first strike stability models, but the loss is minor and reparable. It does contain a non-damage objective that produces gains that can justify conflicts and a value for reducing uncertainty by probing the reward threshold. That probing maps onto the use of exchanges or crises to test resolve in games against opponents, which is the core of the treatment of crisis stability.

Single stage game with given maximum threshold. Consider a game in which the player commits $M$ units of engagement-e.g., progressive levels of political, conventional, or nuclear forces-in the hope of attaining a reward of magnitude $V$. To obtain it, $M$ must exceed some threshold value, which is not known at the outset. If it does, his gain is V - M, i.e., the reward less the effort expended. If not, it is $-\mathrm{M}$. Assume that the threshold is a random variable with a uniform distribution from 0 to some fixed $N$. Then for $M \leq N$, the probability of success is $S=M / N$, the probability of failure $=1-S=1-M / N$, and the expected return is

$$
\begin{aligned}
E V & =\text { Expected gain }+ \text { Expected loss }=S(V-M)+(1-S)(-M) \\
& =M / N(V-M)+(1-M / N)(-M)=(V / N-1) M,
\end{aligned}
$$

which is shown on Fig. 1 for $N=10$ and $V=15$. The expected loss has a minimum at $M=5$ and the expected gain has a maximum at $M=7-8$. The expected value $E V$ increases linearly from 0 to $(15 / 10-1) 10=5$ as $\mathrm{M}$ increases from 0 to 10 .

To maximize EV, the optimal strategy is to choose $M=0$ for $V / N<0$ and $M=10$ for $\mathrm{V} / \mathrm{N}>0$. For $\mathrm{V} / \mathrm{N}=0$ the $\mathrm{EV}=0$ for all $\mathrm{M}$. If steps in $\mathrm{M}$ could be taken sequentially or the game played a number of times, as in a sequence of crises, the player could infer $\mathrm{V}$-and hence the optimal strategy by observing the variation in the gains, losses, and net returns over time.

Single stage game with unknown maximum threshold. Next consider a game in which the player commits $M$ units in the hope of exceeding a threshold that can range up to $N$, which is not known. If he does, his gain is $\mathrm{V}-\mathrm{M}$, and if not, it is $-\mathrm{M}$. If the threshold is a random variable with a uniform distribution from 0 to $N$, the probability of success is $S=\min (M / N, 1)$, the probability of failure $=1-\mathrm{S}$, and the expected value is

$$
\mathrm{EV}=\min (\mathrm{M} / \mathrm{N}, 1)(\mathrm{V}-\mathrm{M})+[1-\min (\mathrm{M} / \mathrm{N}, 1)](-\mathrm{M}) \text {. }
$$

Figure 2 shows the expected gain, whose origin is at the upper right. For $N=1$, the expected gain rises rapidly to a peak at $M=1$, where $S=1$ and $E V=15-1=14$, after which it falls for larger $M$. For larger $N$, the peak drops and shifts to larger $M$, e.g., for $N=10$, it shifts to $M=7$, for which $\mathrm{S}=0.7$, and the expected gain $=0.7 \times 8=5.6$.

Figure 3 shows the expected loss, whose origin is at the lower left. There, the expected loss is small because $M$ is small. For larger $M$, the peak increases and shifts to larger $m$, e.g., for $\mathrm{N}=10$, the peak is at $\mathrm{M}=5$, where $\mathrm{S}=0.5$, and the expected loss $=-0.5 \times 5=2.5$.

Figure 4 shows the expected value EV, whose origin is at the upper right. For $N=1$, the peak at $M=1$ is little reduced from Fig. 2. For larger values of $N$, peak values are reduced and 
shifted to larger $M$, e.g., for $N=9$, the peak is at $M=9$, where $S=1$, and $E V=1 \times 6-0 \times 9=6-0$ $=6$. For larger $\mathrm{N}, \mathrm{M}$ increases monotonically, since the gain has been maximized and the losses continue to compound.

For any given $\mathrm{V}$ and $\mathrm{N}$, there is an optimal $\mathrm{M}$ that maximizes the return to the player. However, the maximum payoff decreases rapidly and shifts to larger values of $\mathrm{M}$ as $\mathrm{N}$ increases, which means that this strategy could take a very large investment and return comparatively little.

Single stage game with threshold estimation. It is often the case that a contestant can gain information about the payoff of a game from the results of a modest or limited number of trials. As an example, consider a game in which each trial reduces the maximum value of the threshold. Then, as long as the initial value has some finite value, each unit will reduce the variance in the threshold, until ultimately the game has a bounded and possibly positive expected value. As a concrete example, assume the initial value of the threshold is $\mathrm{N}$, which is not known to the contestant, and that each unit reduces the maximum value of the threshold by a factor of 2 , so that the $\mathrm{EV}$ after $\mathrm{M}$ units is

$$
\mathrm{EV}=\min \left(\mathrm{M} / \mathrm{N} / 2^{\mathrm{M}}, 1\right)(\mathrm{V}-\mathrm{M})+\left[1-\min \left(\mathrm{M} / \mathrm{N} / 2^{\mathrm{M}}, 1\right)\right](-\mathrm{M}) .
$$

Figure 5 shows the expected gain, loss, and net value as functions of $\mathrm{M}$ for $\mathrm{N}=2^{10}$ and $\mathrm{V}=40$. The top curve is the gain, which rises quadratically with $M$ to a maximum of 12 at $M=25$. The bottom curve is the loss, which initially falls linearly, has a minimum at $M=20$, and returns to zero at $M=30$. Their sum EV first follows the larger losses, passes through 0 at $M=23$, has a maximum of 10 at $M=30$, and falls to zero at $M=40$. For these parameters, the optimum $M$ is 30. Larger values would reduce EV. Smaller values would, too, although $M$ a small as 24 would produce positive EV. However, smaller positive values of $M$ would produce large losses.

There are some resemblance to Fig. 1; however, Fig. 1 is monotonic in M, while Fig. 5 is not. The difference is the estimation of $N$. It reduces the variance for large $M$ even for $N=1,024$, for which Eq. (1) gives probabilities of success $<0.01$ without estimation.

The strong slopes of the losses for small $\mathrm{M}$ might be used to infer $\mathrm{N}$ and $\mathrm{V}$ from the results of small or a small number of trials, but Fig 6, which shows the EV for $V=20,30$, and 40 indicates that the difference is small for $M<10$. Thus, it would be necessary to use $15-20$ units, i.e., about half the optimum value to obtain a reliable estimate of $\mathrm{N}$ and $\mathrm{V}$.

Multi- stage game with unknown threshold. Fig 7 shows the expected gain from Eq. (3) as a function of $\mathrm{M}$ for a range of $\mathrm{N}$ with the origin at the upper right. It resembles the gain of Fig. 2, but the range of $\mathrm{N}$ is much larger because of the estimation of $\mathrm{N}$. The peak at $\mathrm{N}=2$ is at $M=2$. At $N=32$, the peak is at $M=6$, although it is quite small. For larger $N$, it is not visible.

Figure 8 shows the expected losses, which for $N>32$ essentially becomes loss $\sim M$, because the probability of success becomes quite small. 
Figure 9 shows EV, which is negative for $N>4$, but appreciable for smaller $N$ because it is dominated by expected gain at small $\mathrm{N}$ and by loss at large $\mathrm{N}$. The effect of estimation is shown by two trajectories. The first starts from $N=5$, where the optimal $M=0$; through an effective $N=4$, which would be produced by $M=1$; to $N=3$, where $M=2 ; N=2$ and $M=3$; to $N=1$ where the optimal $M=4$. Along this trajectory, the $E V$ increases almost monotonically from 0 at $M=0$, to 0 - at $M=2$, to $\approx 2$ at $M=2$, to 8 at $M=3$, and 11 at $M=4$.

The trajectory starting at $\ln N=10$ is somewhat more extreme; as $N \approx$ would produce essentially zero $E V$ without estimation. As $M$ increases, $E V$ decreases, reaching $\approx-3$ at $M=4$. It then increases, becoming positive at $M \approx 6$. By $M=7$ it reaches $\approx 6$. It then passes over a saddle point and drops down again to $\approx 7$ at $M=8$, decreasing linearly thereafter.

Figure 10 shows the variation of $\mathrm{EV}$ with $\mathrm{M}$ along these estimation-constrained trajectories. The first for $N=1$ peaks at 13. That for $N=5$ increases to join it by $M=4$ where the EV is still 11. That for $N=10$ first decreases and then increases to join at $M=8$. Thus a 30-fold increase in roughly doubles the value of $\mathrm{M}$ at which the trajectories join and reduces the EV at that point by about $\mathrm{M} / 2$. With estimation, these penalties are tolerable.

Comments on connection and extension. The models discussed above do not explicitly contain a number of features that are included in first strike stability models, such as first and second strikes, value and force targets, specific target sets, strike costs, and optimal allocation of weapons. However, for current and projected forces, allocations are relatively stable, so strikes and costs are roughly proportional to weapons. The unit costs included in the models above can be interpreted as the cost of the other's strike as well as the physical cost of the systems, so little is lost by this degree of abstraction. However, if it was necessary to imbed such refinements, they could be included.

What the final model does contain is an explicit objective-other than damage to other. That objective dominates the analysis above. It produces gains that can be large enough to justify conflicts. It also produces a value of such conflicts in reducing uncertainty by sequentially probing the value of the gain threshold and the value of the reward. This value maps into the use of exchanges or crises to test the resolve of the other, when games against an opponent are studied rather than just games against nature, as studied here. Such an extension is the core element of the treatment of crisis stab rather than just first strike stability.

Summary and conclusions. Games against nature illustrate the objective-seeking and uncertainty-reducing activities that are at the core of more detailed treatments of crisis stability. They also illustrate how those processes can generate and deepen crises, as well as the optimal strategies that might be used to end them. They permit the exploration of two essential elements of crisis stability omitted treatments based on first strike stability: an objective that motivates conflicts sufficiently serious to lead to conflicts, and the process of sequential interactions that 
could cause those conflicts to deepen. The model used is a game against nature, simplified sufficiently to make the role of each of those elements obvious.

The simplest single-stage game with a given threshold illustrates the mathematical treatment of the expected gains, losses, and net value. Extension to an unknown maximum threshold shows how the expected value shifts. It illustrates that for any given $V$ and $N$, there is an optimal value of $M$ that maximizes the return to the player, but that the maximum payoff decreases and shifts to larger values of $\mathrm{M}$ as $\mathrm{N}$ increases, which means that this strategy could take a very large investment and return comparatively little

That situation can be improved if the contestant can gain information about the payoff from the results of a limited number of trials. Efficient estimation of the threshold can enable one to cope with threshold uncertainty that would overwhelm non-estimating strategies. The form of the expected loss and gain curves also suggest the possibility of inferring $\mathrm{N}$ and $\mathrm{V}$ from the results of small or a small number of trials, but the model studied here suggests that it might be necessary to use exchanges about half as large as the optima to obtain reliable estimates.

Trajectories starting at large $\mathrm{N}$ produce negative returns until the contestant commits about $\ln N$ units. That reduces the variance in the estimate of $\mathrm{N}$ such that the EV quickly becomes positive. After passing the saddle point, it rejoins the trajectory for small $\mathrm{N}$, albeit at a much lower EV. A 30-fold increase in roughly doubles the value of $M$ at which the trajectories join and reduces the $\mathrm{EV}$ at that point by about $\mathrm{M} / 2$, which is tolerable.

This model does not explicitly contain some features included in first strike stability models, but for current and projected forces, allocations are relatively stable, so the omission is not essential. It does contain are an explicit non-damage, which dominates the analysis. It produces gains that can justify conflicts and value in reducing uncertainty by sequentially probing the values of the gain threshold and reward. both are features that map onto the use of exchanges or crises to test resolve in games against opponents; which is the core of the treatment of crisis stab rather than just first strike stability. 


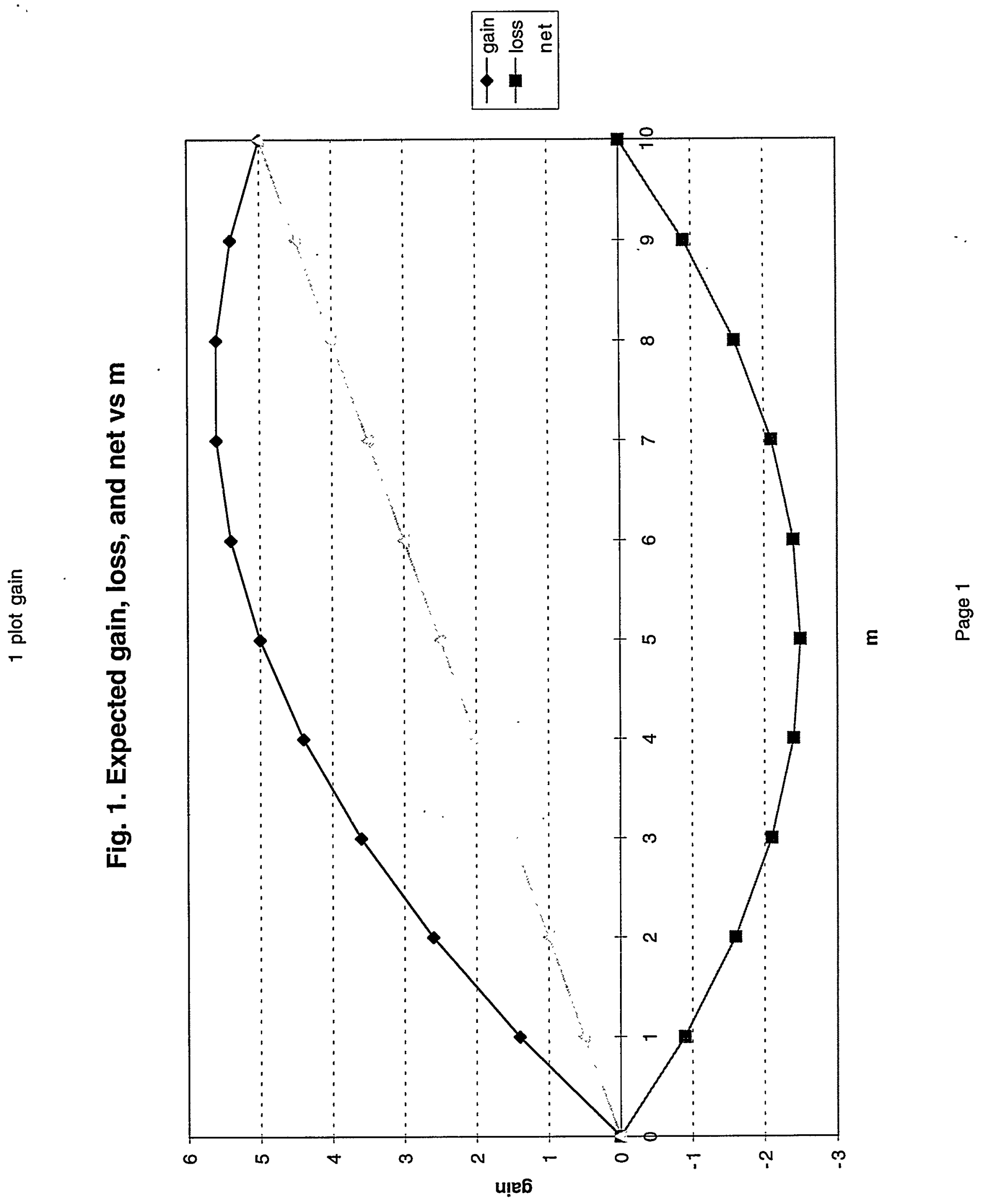




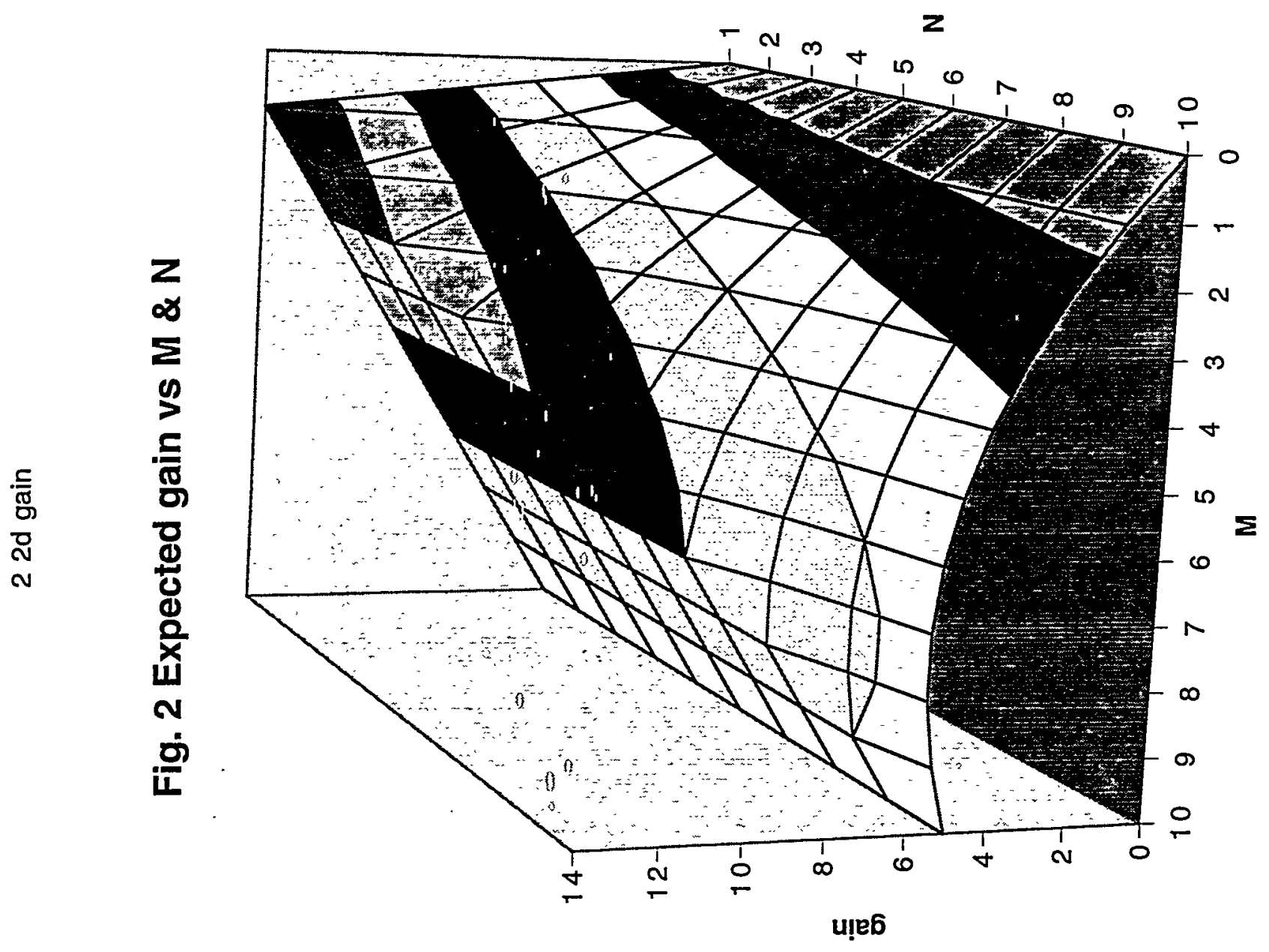

离 


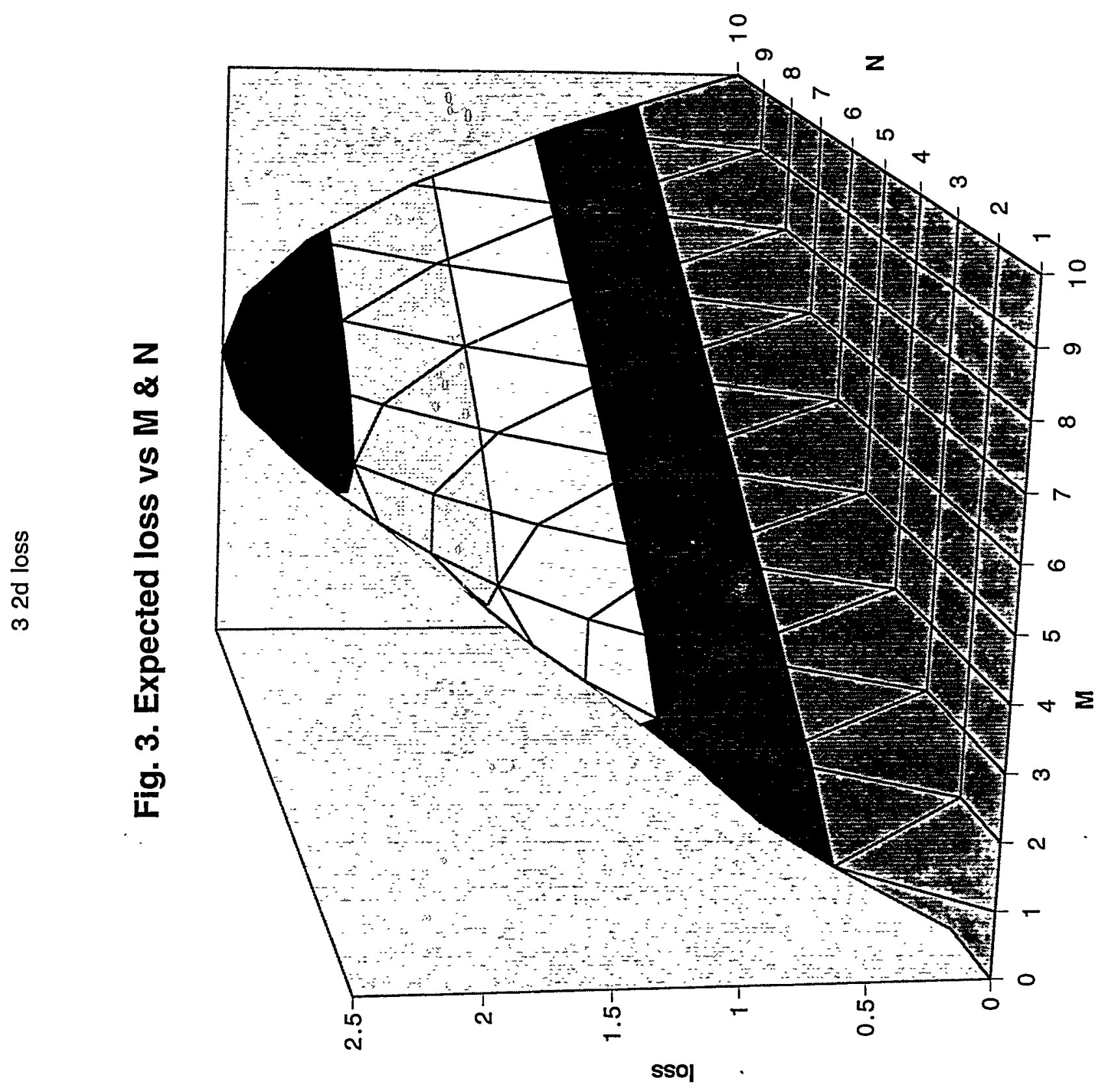

몽 


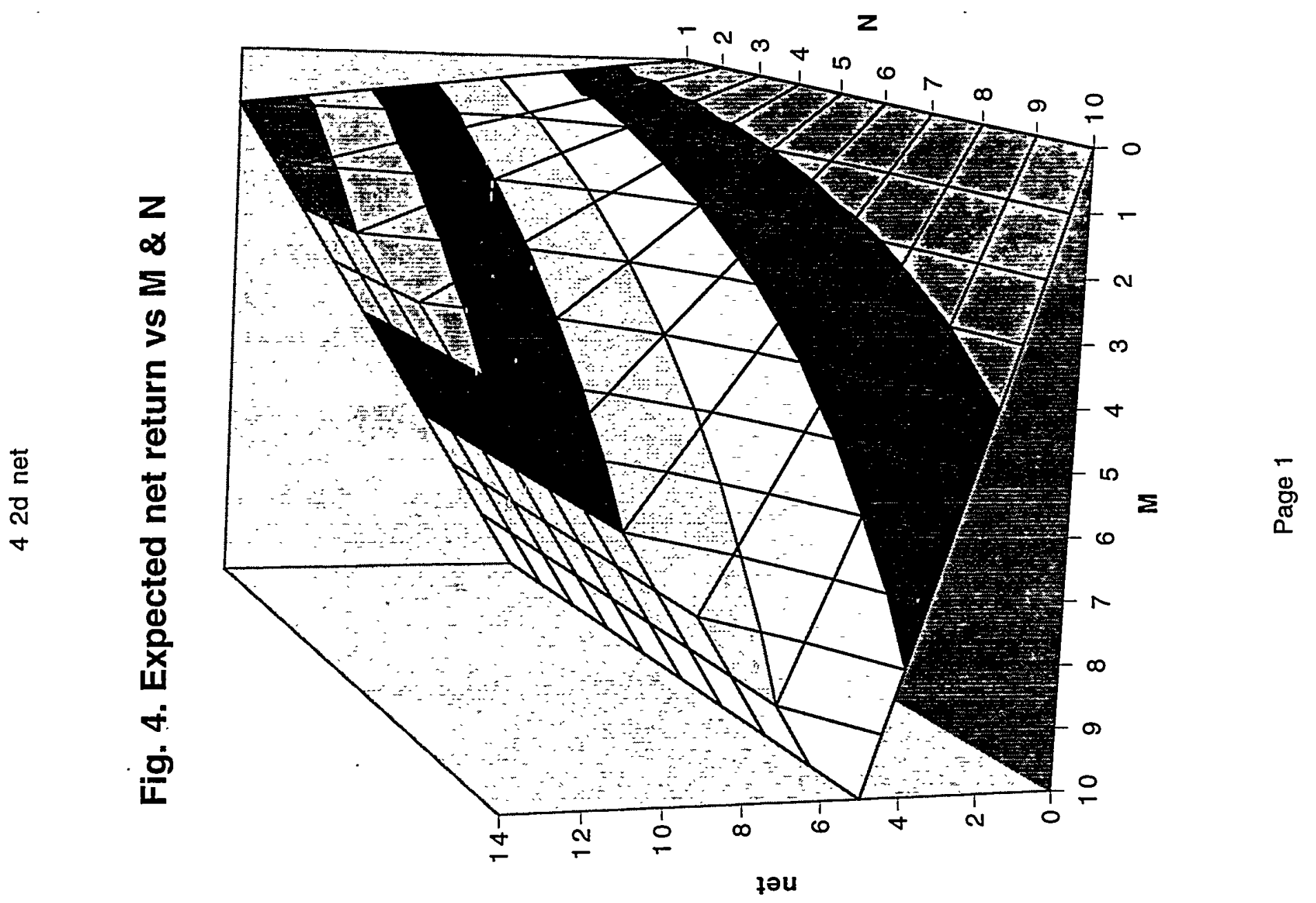




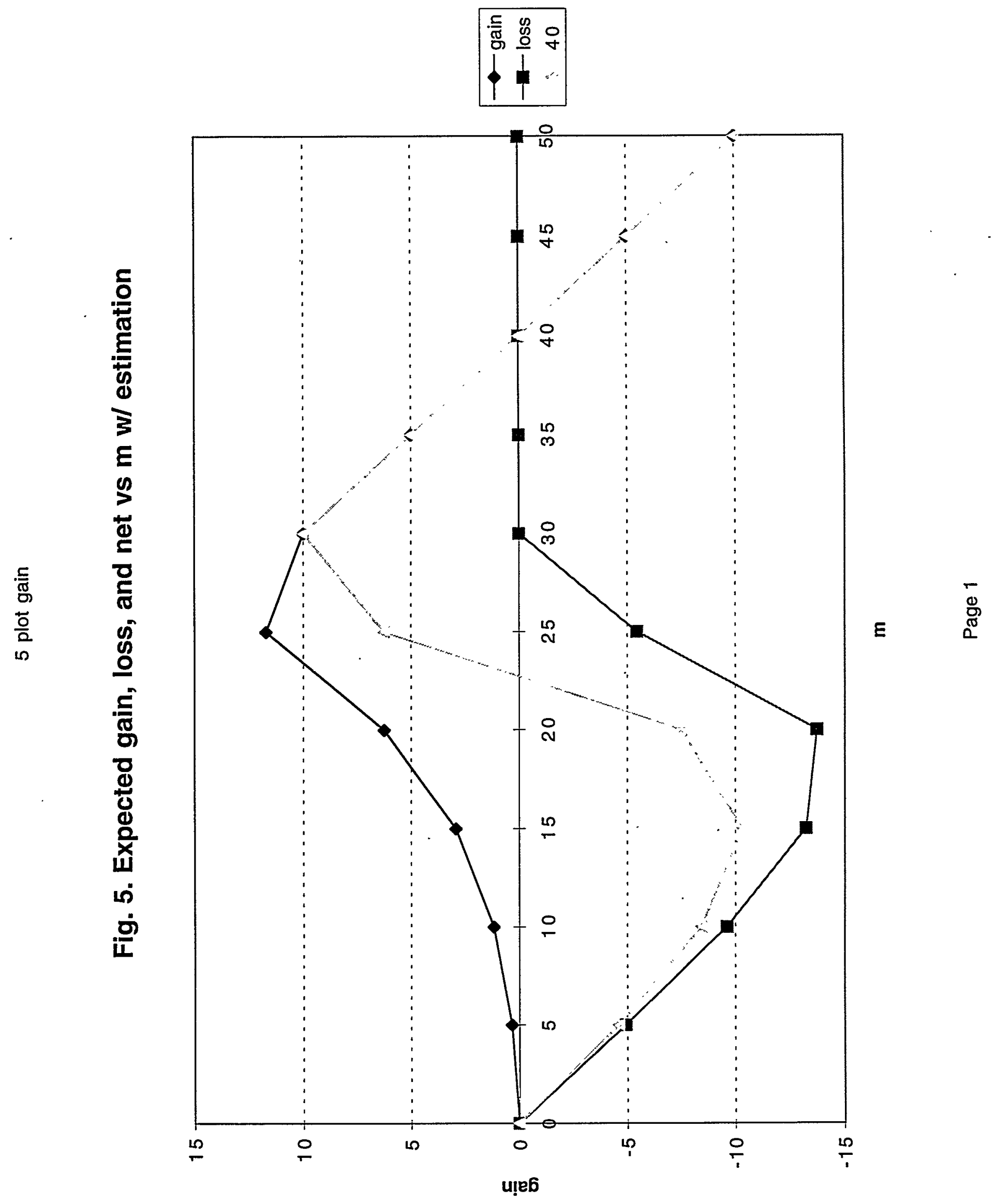




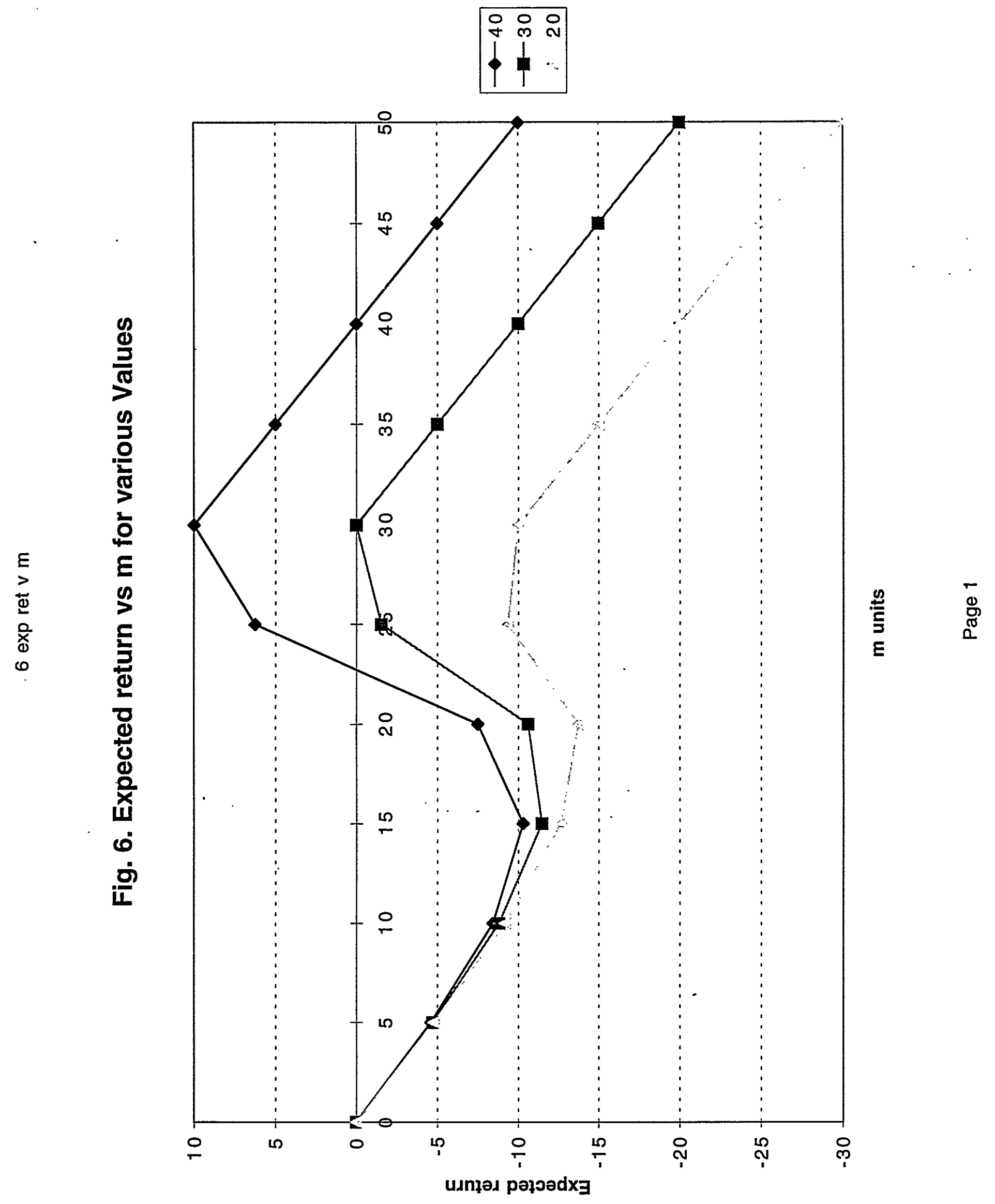




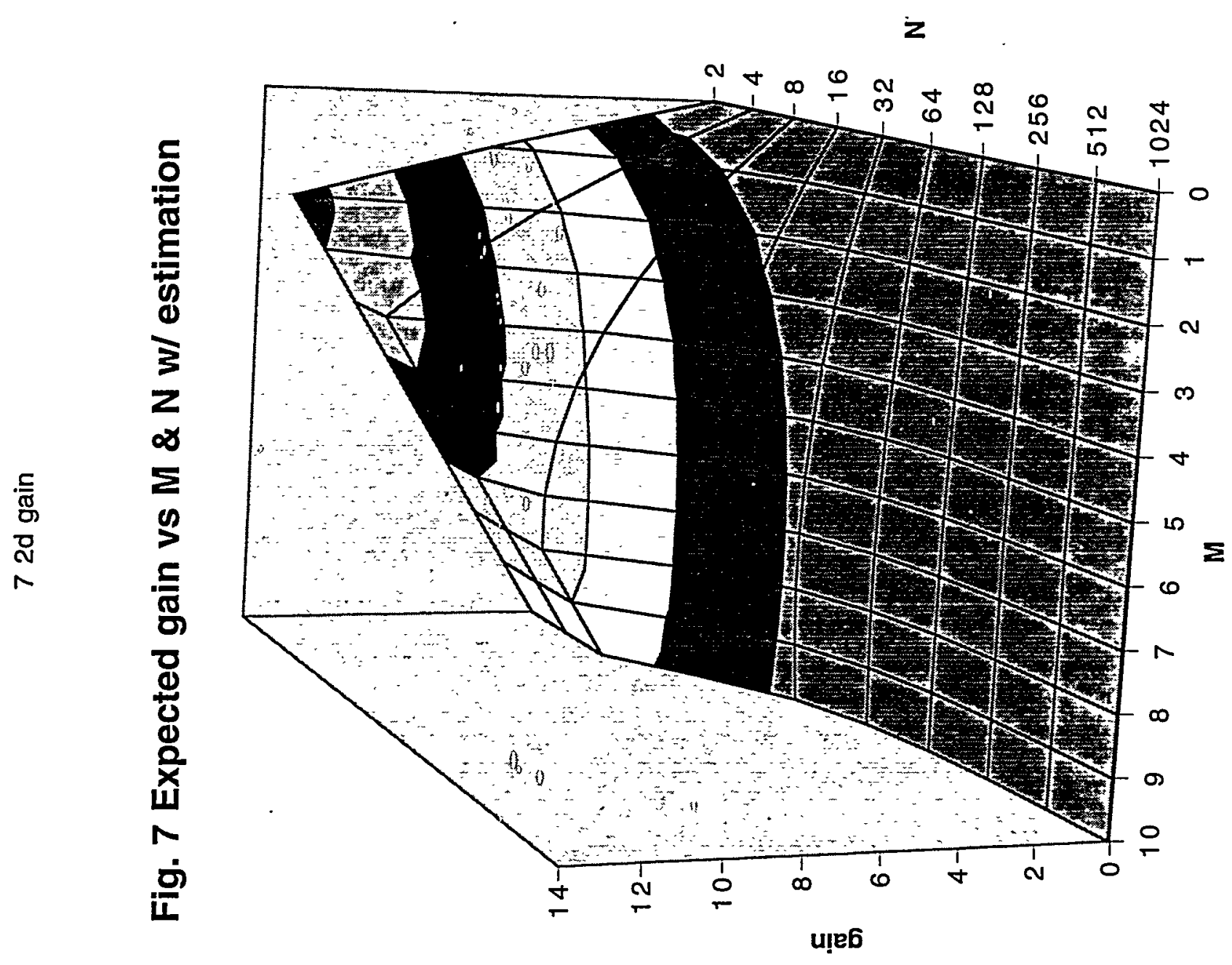

品 
Fig. 8. Expected loss vs $M \& N$ w/ estimation

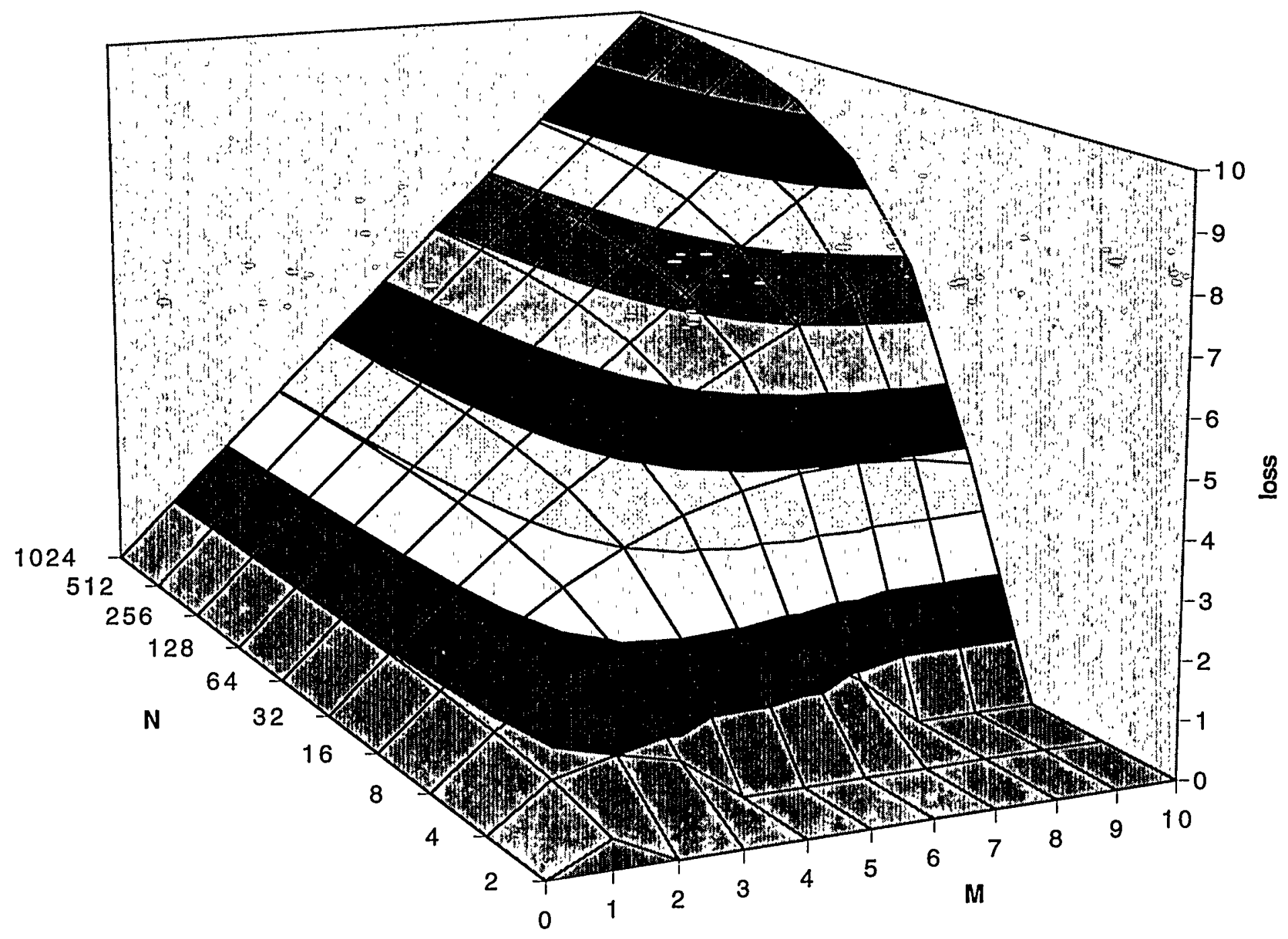




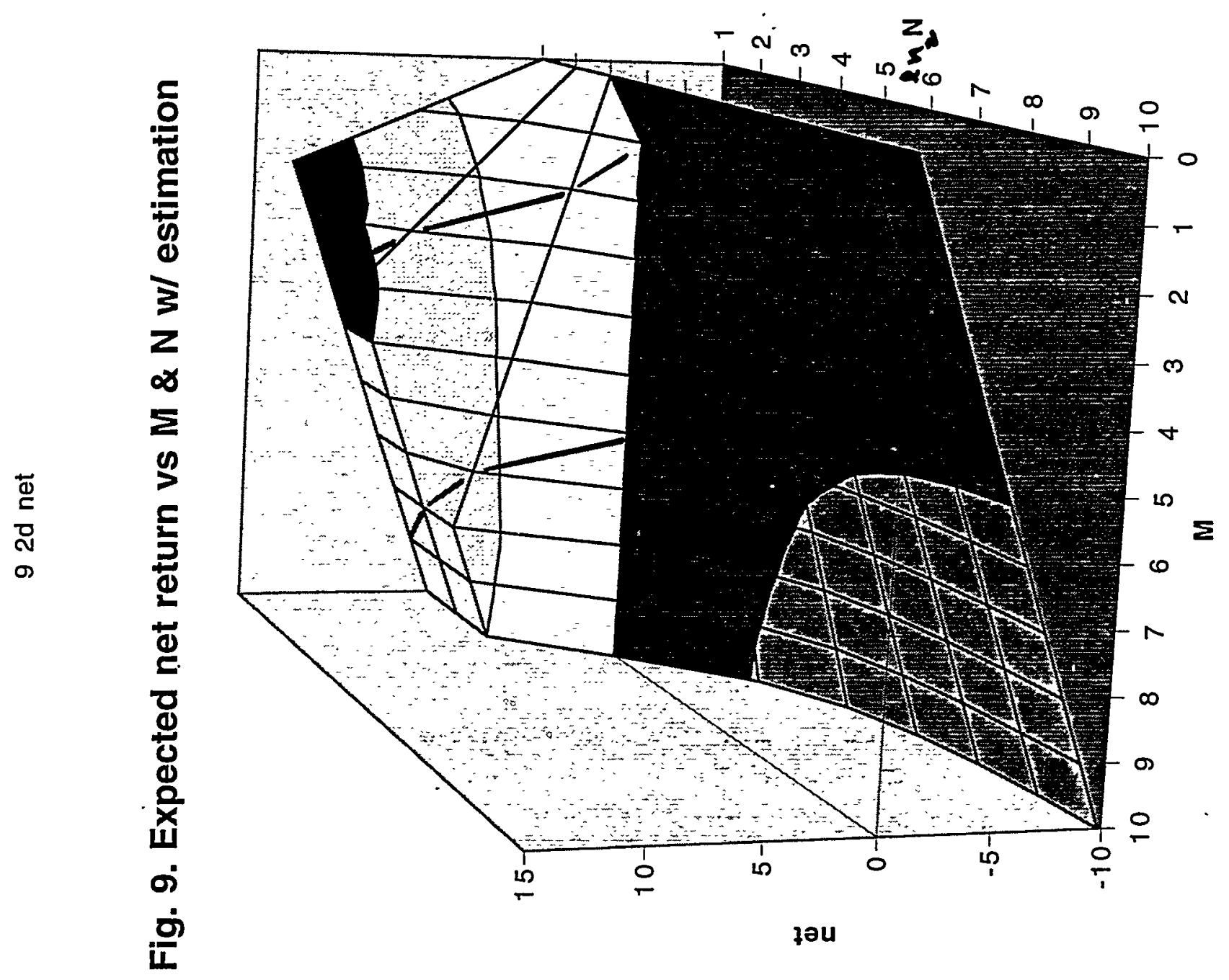

ণ্ণ 


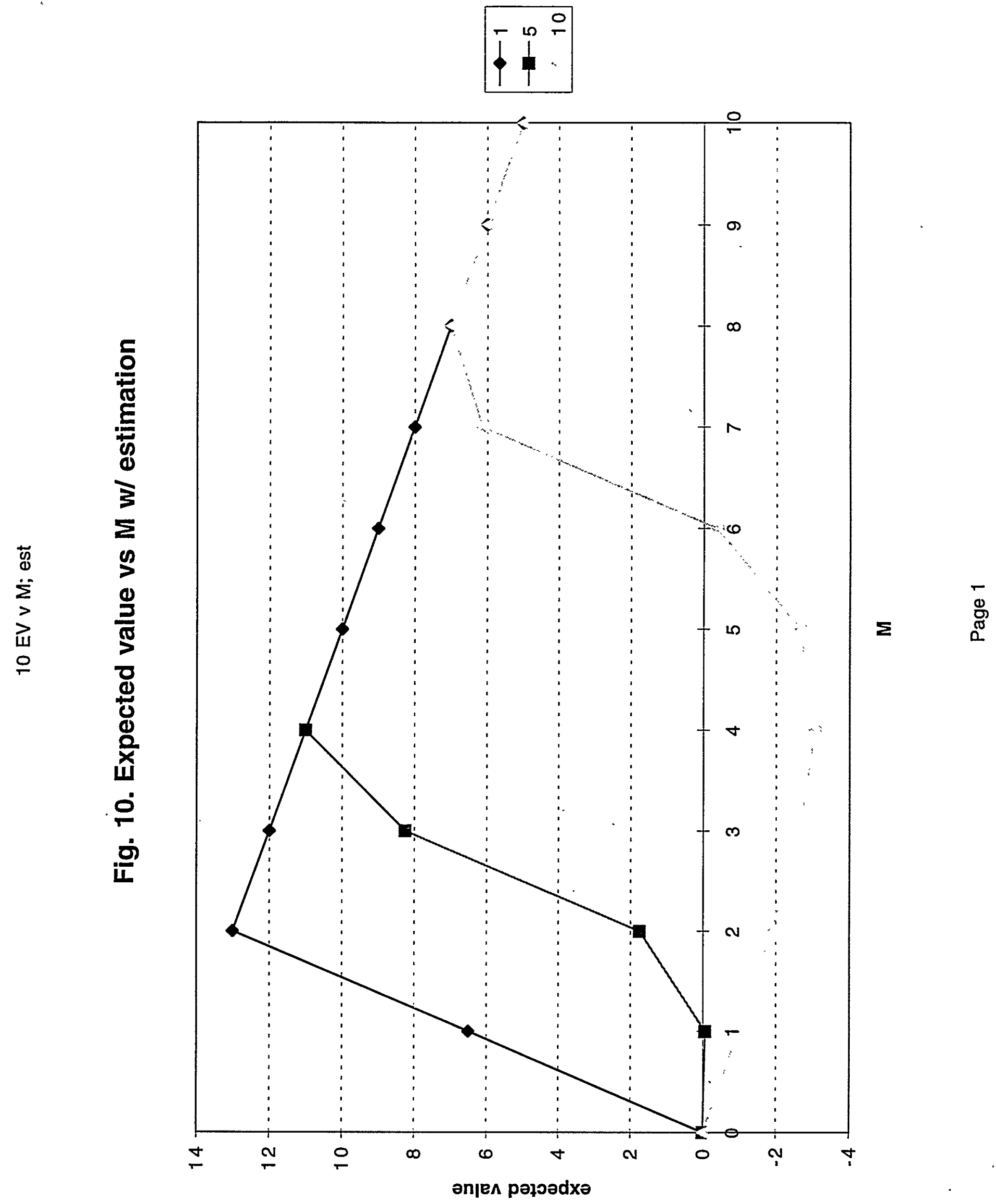

